\title{
Astrophysical dynamos: the limit of vanishing diffusivity
}

\author{
Emmanuel Dormy and Ismaël Bouya
}

\author{
MAG, Ecole Normale Supérieure, 24 rue Lhomond, 75005 Paris \\ email: dormy@phys.ens.fr
}

\begin{abstract}
Astrophysical dynamos are usually characterised by huge values of the magnetic Reynolds number $(\mathrm{Rm})$. This reflects the short turn-over time compared to the resistive time. The extreme values of $\mathrm{Rm}$ relevant to astrophysical objects cannot be tackled with today's numerical resources and this number is always under-estimated by several orders of magnitudes in numerical models.

Here we chose to focus on an extremely simplified problem (dynamo action from a periodic steady flow) and take advantage of this simplicity to numerically investigate the limit of very large magnetic Reynolds number. We present results recently published in physics journals, which highlight the difficulty of approaching the limit in which dynamo action is independent of the value of the ohmic resistivity (measured by $1 / \mathrm{Rm}$ ), known as the "fast dynamo" limit. Using state of the art high performance computing, we present high resolution simulations (up to $4096^{3}$ ) and extend the value of $\mathrm{Rm}$ up to $5 \cdot 10^{5}$.
\end{abstract}

\section{Introduction}

The ABC flow was originally introduced by Arnol'd to investigate Lagrangian chaos. It soon became the prototype example to illustrate fast dynamo action, i.e. dynamo action exhibiting magnetic field amplification on a typical timescale independent of the electrical resistivity of the medium. Even though this flow is the most classical example for this important class of dynamos (with application to large scale astrophysical objects), it was recently pointed out by Bouya and Dormy (2013) that its "fast dynamo" property was still not established.

A "fast dynamo" (see Childress and Gilbert 1995) is a flow which achieves exponential magnetic field amplification over a typical time related to the advective timescale and not the ohmic diffusive timescale (in which case it is referred to as a "slow dynamo"). It is known that exponential stretching of fluid elements is necessary for fast dynamo action. The existence of fast dynamos is essential to account for the presence of magnetic field in astrophysical bodies, for which the ohmic diffusive time is often larger than the age of their formation. If self-excited dynamo action is to generate their magnetic fields, it is therefore essential that it be achieved over an advective timescale.

The most classical flow to exemplify such "fast dynamo" action is indeed the ABC-flow. Arnold and Korkina (1983) first investigated the dynamo property of the ABC-flow. The $\mathrm{ABC}$ flow is a prototype for fast dynamo action, essential to the origin of magnetic field in large astrophysical objects. Probably the most studied configuration is the classical $1: 1: 1$ flow. In a series of two papers published in the physics literature, we investigate its dynamo properties increasing (as much as numerically feasible with today's resources) the magnetic Reynolds number Rm. 


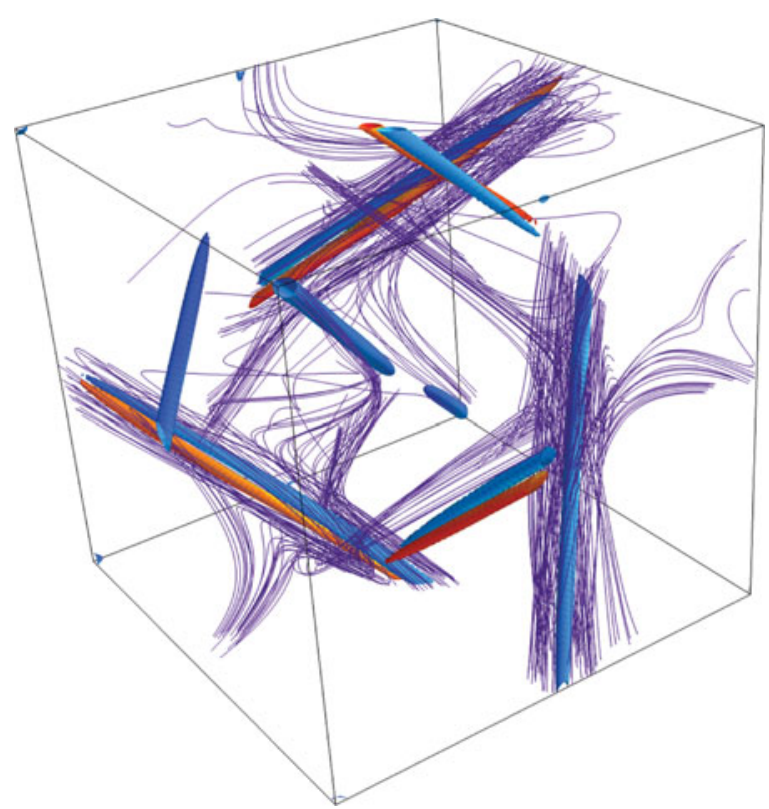

Figure 1. Dynamo eigenmode obtained for $\mathrm{Rm}=434.2$. An isosurface of the magnetic energy is represented. To highlight the symmetry, it is coloured according to the value of $B_{x}$, from blue (negative) to red (positive). The corresponding eigenvalue is purely real, yet the double cigars structure of the field remains clearly visible. Magnetic field lines are also represented (after Bouya and Dormy 2013).

\section{Overview}

We are concerned with the kinematic dynamo problem, for which a solenoidal magnetic field evolution is governed under a prescribed flow by the induction equation

$$
\frac{\partial \mathbf{B}}{\partial t}=\boldsymbol{\nabla} \times\left(\mathbf{u} \times \mathbf{B}-\mathrm{Rm}^{-1} \boldsymbol{\nabla} \times \mathbf{B}\right) .
$$

Finding exponentially growing solutions to this equation is known as the kinematic dynamo problem. We consider here the classical ABC-flow, which takes the form

$$
\mathbf{u}=(A \sin z+C \cos y) \mathbf{e}_{x}+(B \sin x+A \cos z) \mathbf{e}_{y}+(C \sin y+B \cos x) \mathbf{e}_{z},
$$

and restrict our attention to the case where the magnetic field has the same periodicity as the flow (i.e. $2 \pi$-periodic in all directions of space, and the weight of the three symmetric Beltrami components are of equal strength $A: B: C=1: 1: 1$. This choice of coefficients belongs to the largest symmetry class for this kind of flows, and has for this reason been the most intensively studied. We insist that the flow is steady in time and only exhibits Lagrangian chaos.

We have investigated using high resolution direct numerical simulations the behaviour of the 1:1:1 ABC-dynamo, thus extending the work of Galloway and Frisch (1984). The leading eigenmode (fastest growing magnetic field) is represented in figure 1 for $\mathrm{Rm}=434.2$. It gets increasingly localised as $\mathrm{Rm}$ increases. The behaviour of the magnetic field growth-rate $\sigma$ with $\mathrm{Rm}$ is reported on figure 2. We have shown that the two dynamo windows identified for this dynamo are associated with a change of dominant eigenvalue. We have identified a second kink in the growth rate as a function of Rm and shown that it corresponds to an eigenvalue coalescence and the end of the oscillatory nature of the solutions. 


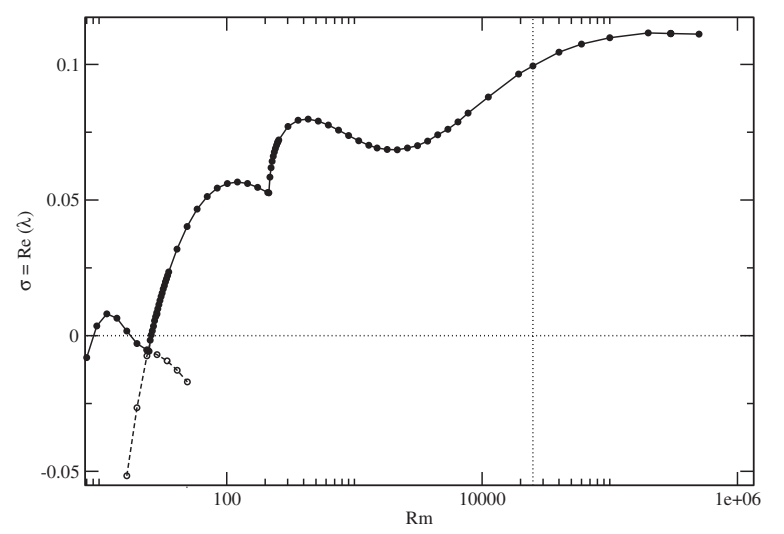

Figure 2. Plot of the growth rate (real part of the leading eigenvalue) as a function of Rm, up to $\mathrm{Rm}=5 \cdot 10^{5}$ (after Bouya and Dormy 2015).

Each simulation we performed involves $N^{3}$ Fourier modes. Simulations up to $\mathrm{Rm}=$ $6 \cdot 10^{4}$ were performed with resolutions $N=512$ and $N=1024$ in order to check convergence. Simulations up to $\mathrm{Rm}=3 \cdot 10^{5}$ were performed with resolutions $N=1024$ and $N=2048$ and the highest $\mathrm{Rm}$ we were able to perform, $\mathrm{Rm}=5 \cdot 10^{5}$ was validated using $N=2048$ and $N=4096$.

Interestingly, even at these huge values of $\mathrm{Rm}$, the growth rate of the leading eigenmode still depends on the controlling parameter and an asymptotic regime is not reached yet. Following an increase of the growth rate over the range $\sim\left[210^{3}, 210^{5}\right]$, we show that the maximum growth rate is a decreasing function of $\mathrm{Rm}$ for the largest values of $\mathrm{Rm}$ we could achieve (as anticipated in Bouya and Dormy 2013). We point the reader to Bouya and Dormy (2015) for an extended discussion.

Several aspects of our simulations seem to indicate that the ABC-dynamo is approaching an asymptotic behaviour for $\mathrm{Rm} \simeq 10^{5}$. We show in, Bouya and Dormy (2015), that the cross sections through the cigar shape structure represented on figure 1 do not reveal any change in structure, once rescaled coordinates are used. This is also supported by the fact that the growthrate is only slightly above the theoretical upper bound and is now decreasing with Rm. The occurrence of damped oscillations points to an approaching eigenvalue. This could be relevant to the asymptotic behaviour, for which an essential spectrum is expected.

However the asymptotic behaviour is not yet established and several issues indicate that one must be cautious in interpreting the numerical results as a "fast dynamo". This stresses the risk of "slow dynamo" action in DNS which usually rely on much more moderate values of the magnetic Reynolds number.

\section{References}

Arnol'd V. I. \& Korkina, E. L. 1983, The growth of a magnetic field in the three-dimensional steady flow of an incompressible fluid, Moskovskii Universitet Vestnik Seriia Matematika Mekhanika, 1, 43.

Bouya, I. \& Dormy, E. 2013, Revisiting the ABC flow dynamo. Physics of Fluids, 25(3).

Bouya, I. \& Dormy, E. 2015, Toward an asymptotic behaviour of the ABC dynamo, EPL, 110, 14003.

Galloway, D. J. \& Frisch, U. 1984, A numerical investigation of magnetic field generation in a flow with chaotic streamlines, Geophysical \&3 Astrophysical Fluid Dynamics, 29, 13.

Childress, S. \& Gilbert, A. D. 1995, Stretch, twist, fold: the fast dynamo, volume 37 of Lecture Notes in Physics Monographs. Springer Berlin Heidelberg. 